\title{
Interleukin-10 -1082 A/G Promoter Polymorphism in Newly Diagnosed Gastro-Intestinal Malignancies and Its Relation to the Nutritional and Performance Status
}

\author{
Somaia M. Mousa ${ }^{1}$, Rowayda A. Mahmoud ${ }^{2}$, Sherif N. Amin ${ }^{1 *}$, Wessam A. El- \\ Sherief ${ }^{3}$
}

${ }^{1}$ Clinical Pathology Department, Kasr Al-Ainy School of Medicine, Cairo University, Cairo, Egypt; ${ }^{2}$ National Nutrition Institute, The General Organization for Teaching Hospitals and Institutes, Ministry of Health, Cairo, Egypt; ${ }^{3}$ Clinical Oncology Department, Kasr Al-Ainy School of Medicine, Cairo University, Cairo, Egypt

\begin{abstract}
Background: Cancer is associated with a state of systemic inflammation with high levels of circulating inflammatory cytokines. Cytokine gene polymorphisms may affect the inflammatory response in cancer patients and their nutritional and performance status with subsequent effects on their prognosis.

Aim: This study investigated the relationship between IL-10 -1082 A/G promoter polymorphism and anorexia, body mass index (BMI) and performance status in patients with gastrointestinal (GI) malignancies.

Methods 88 newly diagnosed patients with GI malignancies were genotyped for IL-10-1082 A/G promoter polymorphism by real-time PCR allelic discrimination assay. The prevalence of anorexia, BMI and performance status was compared between patients with different genotypes.

Results: In the whole group of patients, anorexia was significantly more prevalent $(\mathrm{p}<0.001)$ among patients carrying the variant genotypes ( $A G$ and $G G$ ) who were also found to have significantly lower BMI ( $p=0.002$ ) and poorer performance status $(\mathrm{p}=0.027)$ than those with the wild genotype (AA). In patients with earlier cancer (stages I and II), a variant genotype was significantly associated with more anorexia and lower BMI.

Conclusion: These results suggest that IL-10-1082 A/G promoter polymorphism may have an effect on the nutritional and performance status of patients with GI malignancies.

Keywords: Anorexia, Body mass index, Cancer, Cytokines, IL-10 polymorphism, Performance status Corresponding Author: Somaia Mousa, MD; Clinical Pathology Department, Kasr Al-Ainy School of Medicine, Cairo University. Cairo, Egypt; Email: somaiamousa@kasralainy.edu.eg

Submitted: 29-January-2021, Finally revised: 21-February-2021, Accepted: 1-March-2021, Published online: 1-April-2021
\end{abstract}

(cc) BY

*In memoriam (1961-2017)

\section{Introduction}

The inflammatory and nutritional status of patients with gastrointestinal malignancies and their performance status have a significant impact on their short- as well as long-term outcomes ${ }^{1-6}$.

Nutritional and immune-inflammatory status of patients with gastric cancer are important in recognizing high-risk patients and predicting overall and disease-free survival ${ }^{1}$. Patients with poor nutritional status have a higher risk of cancer and non-cancer related mortality ${ }^{2}$. Markers of nutritional status like body mass index (BMI), prognostic nutritional index and preoperative weight loss are important prognostic indicators in patients with gastrointestinal (GI) malignancies ${ }^{3,4,5}$. Gastric cancer patients with low BMI $(<25)$ were found to have severe postoperative complications, poor survival and poor prognosis ${ }^{6}$. Underweight patients with colon cancer have a worse prognosis compared to those with normal BMI at diagnosis ${ }^{7}$. Involuntary weight loss in patients with GI malignancies is attributed to several factors like systemic inflammation and poor dietary intake ${ }^{8}$. Exaggerated weight loss due to systemic 
inflammatory response to cancer is associated with deterioration of patient's performance status ${ }^{9}$.

Studies indicated that there is a relation between several pro-inflammatory cytokines like tumour necrosis factor- $\alpha$, interferon- $\gamma$, interleukin (IL)-1, IL6 , and IL-10 with weight loss and anorexia in patients with GI cancer ${ }^{9,10}$. Interleukin-10 has a dual effect on the immune response against cancer ${ }^{11}$. Its level was found to be significantly high in sera of patients with cancer cachexia ${ }^{12,13}$. Since the rate of release of inflammatory cytokines is genetically determined, polymorphisms in cytokine genes may explain the variations among cancer patients in the rate and severity of weight loss and may help in predicting patients susceptible to an exaggerated inflammatory response with subsequent weight loss in response to cancer ${ }^{14}$.

The aim of this work was to study the relationship between IL-10-1082 promoter polymorphism and anorexia, BMI and performance status in newly diagnosed patients with GI malignancies.

\section{Methods}

\section{Study population}

This was an observational cross-sectional study that included newly diagnosed patients with GI cancer who visited Kasr Al-Ainy Centre of Clinical Oncology and Nuclear Medicine (NEMROC) during the year 2014.

In order to determine the presence of anorexia, we used the appetite item of the European Organization for Research and Treatment of Cancer Core Quality of Life Questionnaire (EORTC QLQ) C30 version $3.0^{15}$. Patients were asked "Have you lacked appetite?" and the response was rated on a 1 to 4 -point Likert scale $(1=$ not at all and $4=$ very much).

Patients' weight and height were measured and BMI was calculated (BMI=weight in kilogram/ height ${ }^{2}$ in meter). Underweight was defined as a $\mathrm{BMI}<20$, a cutoff value used in the definition of cancer cachexia ${ }^{16}$. Patient's performance status was assessed using the Karnofsky performance status (KPS) score ${ }^{17}$.

\section{Genotyping}

Detection of IL-10-1082 A/G gene promoter polymorphism (rs1800896) was done by real-time PCR allelic discrimination assay using Taq-Man ${ }^{\circledR}$ SNP Genotyping Assays (Applied Biosystems, Foster City, CA, USA) as described before ${ }^{18}$. DNA extraction was done using Thermo Scientific GeneJET Whole
Blood Genomic DNA Purification Mini Kit. The sequence-specific forward and reverse primers used to amplify the promoter region of IL-10 gene are the following:

Forward primers:

5'-CCAAGACAACACTACTAAGGCTTCT-3'

Reverse primers:

5'-GCTGGATAGGAGGTCCCTTACTTT-3'

Two TaqMan ${ }^{\circledR}$ minor groove binder (MGB) fluorogenic probes with different fluorescence dyes were used to distinguish the two alleles. One probe labelled with VIC dye to detect the wild allele (A) CCCTACTTCCCCTTCCCAA-VIC and another probe labelled with FAM dye to detect the variant allele (G) CCTACTTCССССТСССАA- FAM.

The PCR conditions consisted of an initial step of $95^{\circ} \mathrm{C}$ for $10 \mathrm{~min}$ for AmpliTaq Gold activation, followed by 50 cycles of $92^{\circ} \mathrm{C}$ for 15 seconds and $60^{\circ} \mathrm{C}$ for $1 \mathrm{~min}$.

Analysis was performed using Sequence Detection System (SDS), version 2.0, software. The plotted fluorescence signals indicate the types of alleles in each sample. Detection of VIC dye fluorescence only indicates a wild genotype, of FAM dye fluorescence only indicates a homozygous variant genotype and of both indicates a heterozygous genotype.

\section{Statistical analysis}

Numerical data were tested for normality using the Shapiro-Wilk test. The significance of differences in abnormally-distributed numerical data between two independent samples was tested using the Mann-Whitney test.

Qualitative data were expressed as frequency and percentage and were compared using Chi square $\left(\chi^{2}\right)$ test. Fisher's exact test was used instead when the expected frequency is less than 5 . P values less than 0.05 was considered statistically significant.

Data were analysed using Statistical Package for Social Science (SPSS) (SPSS Inc., Chicago, IL, USA) version 15.

\section{Ethical considerations}

The study was approved by the Ethical Committee of Kasr Al-Ainy School of Medicine, Cairo University and patients provided informed consent.

\section{Results}

The study included 88 patients with newly diagnosed gastrointestinal malignancies. Their 
characteristics are shown in Table 1 . The most common primary cancer was colorectal. Sixty-two (70.5\%) patients had anorexia (a loss of appetite score of 2 to 4). Genotyping of IL-10 -1082 polymorphism revealed that $30(34 \%)$ patients had the wild (AA) genotype and 58 (66\%) had the heterozygous (AG) / homozygous (GG) variant genotype. The prevalence of wild-allele (A) was $83 \%$ and that of variant allele (G) $66 \%$.

Table 1: Patients' characteristics $(n=88)$

\begin{tabular}{|c|c|c|}
\hline Variable & Description & \\
\hline Age (years) & Median (IQR) & $\begin{array}{l}47.5(39- \\
59.5)\end{array}$ \\
\hline \multicolumn{3}{|l|}{ Gender } \\
\hline Female & $n(\%)$ & 49 (55.7) \\
\hline Male & $n(\%)$ & $39(44.3)$ \\
\hline \multicolumn{3}{|l|}{ Primary cancer } \\
\hline Colorectal & $n(\%)$ & $68(77.3)$ \\
\hline Stomach & $n(\%)$ & $11(12.5)$ \\
\hline Pancreas & $n(\%)$ & $7(8)$ \\
\hline Esophagus & $n(\%)$ & $2(2.3)$ \\
\hline \multicolumn{3}{|l|}{ Cancer stage } \\
\hline I & $n(\%)$ & $10(11.4)$ \\
\hline II & $n(\%)$ & $23(26.1)$ \\
\hline III & $n(\%)$ & 35 (39.8) \\
\hline IV & $n(\%)$ & $20(22.7)$ \\
\hline \multicolumn{3}{|l|}{$\begin{array}{l}\text { EORTC QLQ-C30 } \\
\text { appetite item score }\end{array}$} \\
\hline 1 & $n(\%)$ & $26(29.5)$ \\
\hline $2-4$ & $n(\%)$ & $62(70.5)$ \\
\hline BMI $\left(\mathrm{kg} / \mathrm{m}^{2}\right)$ & Median (IQR) & $\begin{array}{l}22.5(19.6- \\
25)\end{array}$ \\
\hline KPS score & Median (IQR) & $70(70-80)$ \\
\hline \multicolumn{3}{|l|}{ IL10 -1082 genotype } \\
\hline Wild (AA) & $n(\%)$ & $30(34.1)$ \\
\hline $\begin{array}{l}\text { Heterozygous variant } \\
\text { (AG) }\end{array}$ & $n(\%)$ & $43(48.9)$ \\
\hline $\begin{array}{l}\text { Homozygous variant } \\
\text { (GG) }\end{array}$ & $n(\%)$ & $15(17)$ \\
\hline
\end{tabular}

IQR: Interquartile range, EORTC QLQ: European Organization for Research and Treatment of Cancer Core Quality of Life Questionnaire, BMI: Body mass index, KPS: Karnofsky performance status

Anorexia (EORTC QLQ-C30 appetite score 2 to 4) was significantly more prevalent among patients with the variant genotypes who had a significantly lower performance status score as well (Table 2). Patients with the variant genotype had a significantly lower BMI and were more likely to be underweight.
Twenty-three (26.1\%) of patients had both anorexia of any grade combined with underweight, 39 (44.3\%) had anorexia without underweight and 26 (29.5\%) had no anorexia nor underweight. The majority $(20 / 23,87 \%)$ of patients presenting with anorexia combined with underweight carried a variant genotype. On the other hand, a minority $(10 / 16,38.5 \%)$ of patients without anorexia or underweight carried it. The difference in prevalence between the two groups was highly significant ( $\mathrm{p}<$ 0.001).

A subgroup analysis according to the stage of the disease was performed (Table 3). In patients with earlier stages (I and II) cancer, the prevalence of a variant genotype was significantly higher among patients with anorexia. Also, in earlier cancer patients, the BMI was significantly lower in those with a variant genotype.

\section{Discussion}

This study investigated the possible association between IL-10 - 1082 polymorphism and anorexia, BMI, and performance status in newly diagnosed patients with GI malignancies.

The prevalence of weight loss among patients with upper GI cancer at the time of diagnosis was found to be $80 \%{ }^{19}$. Weight loss in patients with GI cancers is attributed not only to dysphagia, nausea and vomiting associated with such tumours but also other factors are involved like the co-existing state of systemic inflammation ${ }^{8}$. The level of acute phase proteins correlates with the rate of weight loss in patients with GI cancer ${ }^{8}$. Cytokine gene polymorphisms can influence weight loss through changing levels of cytokine production. In the present study, anorexia, low BMI and poor performance status were more prevalent among patients with GI malignancies who carry the variant genotypes (AG and GG) of IL-10 -1082. In concordance with our study, IL-10 -1082G allele has been found to be significantly associated with weight loss in three previous studies that included patients with gastroesophageal and gastric cancers 12, 20, 21. Anorexia, underweight and poorer performance status may be explained by an exaggerated systemic inflammatory response associated with the presence of the variant genotypes of IL-10 -1082 polymorphism. IL-10 polymorphism at position -1082 is present within the promoter region of the gene and the variantgenotypes have been shown to be associated with 
Table 2: Comparing variables according to IL-10 polymorphism

\begin{tabular}{|c|c|c|c|c|}
\hline Variable & & $\begin{array}{l}\text { Wild genotype, AA } \\
(n=30)\end{array}$ & $\begin{array}{l}\text { Variant genotypes, AG/GG } \\
(n=58)\end{array}$ & $p$ value \\
\hline Age (years) & Median (IQR) & $49.5(39-58)$ & $47(39-60)$ & 0.712 \\
\hline \multicolumn{5}{|l|}{ Cancer stage } \\
\hline I-II & $n(\%)$ & $13(39.4)$ & $20(60.6)$ & \multirow[t]{2}{*}{0.416} \\
\hline III-IV & $n(\%)$ & $17(30.9)$ & $38(69.1)$ & \\
\hline \multicolumn{5}{|c|}{ EORTC-QLQ - C30 appetite item score } \\
\hline 1 & $n(\%)$ & $16(61.5)$ & $10(38.5)$ & \multirow[t]{2}{*}{$<0.001$} \\
\hline $2-4$ & $n(\%)$ & $14(22.6)$ & $48(77.4)$ & \\
\hline BMI $\left(\mathrm{kg} / \mathrm{m}^{2}\right)$ & Median (IQR) & $24(21.7-27)$ & $21(19.5-24)$ & 0.002 \\
\hline \multicolumn{5}{|c|}{ Underweight (BMI <20) } \\
\hline No & $n(\%)$ & $27(90)$ & $38(65.5)$ & \multirow[t]{2}{*}{0.02} \\
\hline Yes & $n(\%)$ & $3(10)$ & $20(34.5)$ & \\
\hline KPS score & Median (IQR) & $80(70-80)$ & $70(70-80)$ & 0.027 \\
\hline
\end{tabular}

IQR: Interquartile range, EORTC QLQ: European Organization for Research and Treatment of Cancer Core Quality of Life Questionnaire, BMI: Body mass index, KPS: Karnofsky performance status

Table 3: Subgroup analysis according to cancer stage comparing patients with wild genotype to those with a variant genotype

\begin{tabular}{|c|c|c|c|c|}
\hline Variable & & $\begin{array}{l}\text { Wild genotype, } \\
\text { AA }\end{array}$ & $\begin{array}{l}\text { Variant genotypes, } \\
\text { AG/GG }\end{array}$ & $p$ value \\
\hline \multicolumn{5}{|c|}{ Cancer stage I-II group $(n=33)$} \\
\hline \multicolumn{5}{|c|}{ EORTC QLQ - C30 appetite item score } \\
\hline 1 & $n(\%)$ & $11(64.7)$ & $6(35.3)$ & \multirow[t]{2}{*}{0.004} \\
\hline $2-4$ & $n(\%)$ & $2(12.5)$ & $14(87.5)$ & \\
\hline BMI $\left(\mathrm{kg} / \mathrm{m}^{2}\right)$ & Median (IQR) & $25.9(23.3-27.4)$ & $22.7(19.8-25)$ & 0.039 \\
\hline \multicolumn{5}{|c|}{ Underweight (BMI <20) } \\
\hline No & $n(\%)$ & $12(44.4)$ & $15(55.6)$ & \multirow[t]{2}{*}{0.364} \\
\hline Yes & $n(\%)$ & $1(16.7)$ & $5(83.3)$ & \\
\hline KPS score & Median (IQR) & $80(80-80)$ & $80(70-80)$ & 0.094 \\
\hline
\end{tabular}

Cancer stage III-IV group ( $\mathrm{n}=55)$

EORTC QLQ - C30 appetite item score

\begin{tabular}{|c|c|c|c|c|}
\hline 1 & $n(\%)$ & $5(55.6)$ & $4(44.4)$ & 0.116 \\
\hline $2-4$ & $n(\%)$ & $12(26.1)$ & $34(73.9)$ & \\
\hline BMI $\left(\mathrm{kg} / \mathrm{m}^{2}\right)$ & Median (IQR) & $24(20-26.7)$ & $20.65(18.7-23)$ & 0.029 \\
\hline \multicolumn{5}{|c|}{ Underweight (BMI <20) } \\
\hline No & $n(\%)$ & 15 (39.5) & $23(60.5)$ & \multirow[t]{2}{*}{0.058} \\
\hline Yes & $n(\%)$ & $2(11.8)$ & $15(88.2)$ & \\
\hline KPS score & Median (IQR) & $70(70-80)$ & $70(70-80)$ & 0.182 \\
\hline
\end{tabular}

IQR: Interquartile range, EORTC QLQ: European Organization for Research and Treatment of Cancer Core Quality of Life Questionnaire, BMI: Body mass index, KPS: Karnofsky performance status

increased IL-10 production that was attributed to a higher affinity of the $\mathrm{G}$ allele for Sp1 transcription factor with subsequent higher expression and IL-10 secretion ${ }^{22}$. Moreover, IL-10 -1082GG genotype was found to be associated with elevated markers of systemic inflammation like CRP ${ }^{23}$.

A limitation to this study is that the relationship between IL-10 -1082 A/G promoter polymorphism and the degree of weight loss was not assessed. This 
is because of the absence of accurate patient weight measurements before presenting with cancer. Other limitations included the conduction of the study in a single center, the inclusion of a heterogeneous group of malignancies without equal representation and the relatively small sample size.

\section{Conclusion}

IL-10-1082 variant genotypes (AG and GG) may have an effect on the nutritional and performance status of patients with GI cancer.

Recognizing the cause and effect of systemic inflammation on cancer patients' nutritional and performance status can help to develop proper therapeutic strategies to prevent unnecessary morbidities and to improve patients' response to therapy.

\section{Acknowledgment \\ None. \\ Authors' contribution \\ Conception or design: All authors; Acquisition, analysis or interpretation of data: SMM \& RAM; Drafting the manuscript: SMM \& RAM; Revising the manuscript: WAE; Approval of the manuscript version to be published: SMM, RAM \& WAE; Agreement to be accountable for all aspects of the work: SMM, RAM \& WAE.}

\section{Conflict of interest}

The authors declare that they have no conflict of interest to disclose.

\section{Data availability}

Deidentified individual participant data used to produce the results of this study are available from the corresponding author (SMM) upon request.

\section{Funding}

This research did not receive any specific grant from funding agencies in the public, commercial, or not-for-profit sectors

\section{Study registration}

None.

\section{References}

1. Galizia G, Auricchio A, de Vita F, et al. Inflammatory and nutritional status is a predictor of long-term outcome in patients undergoing surgery for gastric cancer. Validation of the Naples prognostic score. Ann Ital Chir. 2019; 90: 404-416.

2. Migita K, Takayama T, Saeki K, et al. The prognostic nutritional index predicts long-term outcomes of gastric cancer patients independent of tumor stage. Ann Surg Oncol. 2013; 20(8): 2647-2654.

3. Zhang SS, Yang H, Luo KJ, et al. The impact of body mass index on complication and survival in resected oesophageal cancer: a clinical-based cohort and meta-analysis. Br J Cancer. 2013;109(11): 2894-2903.
4. van der Schaaf MK, Tilanus HW, van Lanschot JJ, et al. The influence of preoperative weight loss on the postoperative course after esophageal cancer resection. J Thorac Cardiovasc Surg. 2014; 147(1): 490495.

5. Jiang N, Deng JY, Ding XW, et al. Prognostic nutritional index predicts postoperative complications and long-term outcomes of gastric cancer. World J Gastroenterol. 2014; 20(30): 1053710544.

6. Chen HN, Chen XZ, Zhang WH, et al. The impact of body mass index on the surgical outcomes of patients with gastric cancer: A 10-year, single-institution cohort study. Medicine (Baltimore). 2015; 94(42): e1769.

7. Sinicrope FA, Foster NR, Yothers G, et al. Body mass index at diagnosis and survival among colon cancer patients enrolled in clinical trials of adjuvant chemotherapy. Cancer. 2013; 119(8): 1528-1536.

8. Deans DA, Tan BH, Wigmore SJ, et al. The influence of systemic inflammation, dietary intake and stage of disease on rate of weight loss in patients with gastrooesophageal cancer. Br J Cancer. 2009; 100(1): 63-69.

9. O'Gorman P, McMillan DC, McArdle CS. Longitudinal study of weight, appetite, performance status and inflammation in advanced gastrointestinal cancer. Nutr Cancer. 1999; 35(2): 127-130.

10. Anandavadivelan P, Lagergren P. Cachexia in patients with oesophageal cancer. Nat Rev Clin Oncol. 2016; 13(3): 185-198.

11. Mocellin S, Marincola FM, Young HA. Interleukin-10 and the immune response against cancer: a counterpoint. J Leukoc Biol. 2005; 78(5): 1043-1051.

12. Sun F, Sun $\mathrm{Y}, \mathrm{Yu} \mathrm{Z}$, et al. Interleukin-10 gene polymorphisms influence susceptibility to cachexia in patients with low-third gastric cancer in a Chinese population. Mol Diagn Ther. 2010; 14(2): 95-100.

13. Tazaki E, Shimizu N, Tanaka R, et al. Serum cytokine profiles in patients with prostate carcinoma. Exp Ther Med. 2011; 2(5): 887-891.

14. Tan BH, Ross JA, Kaasa S, Skorpen F, Fearon KC; European Palliative Care Research Collaborative. Identification of possible genetic polymorphisms involved in cancer cachexia: a systematic review. J Genet. 2011; 90(1): 165-177.

15. The European Organization for Research and Treatment of Cancer Core Quality of Life Questionnaire (EORTC QLQ) - C30, version 3.0. Available from: https://qol.eortc.org/questionnaire/eortcqlq-c30/

16. Fearon K, Strasser F, Anker SD, et al. Definition and classification of cancer cachexia: an international consensus. Lancet Oncol. 2011; 12: 489-495.

17. Karnofsky DA, Abelmann WH, Craver LF, Burchenal $\mathrm{JH}$. The use of the nitrogen mustards in the palliative treatment of carcinoma - with particular reference to bronchogenic carcinoma. Cancer. 1948; 1(4): 634-656.

18. Brouard J, Knauer N, Boelle PY, et al. Influence of interleukin-10 on Aspergillus fumigatus infection in 
patients with cystic fibrosis. J Infect Dis. 2005; 191(11): 1988-1991.

19. Bruera E. ABC of palliative care. Anorexia, cachexia, and nutrition. BMJ. 1997; 315(7117): 1219-1222.

20. Deans DA, Tan BH, Ross JA, et al. Cancer cachexia is associated with the IL10 -1082 gene promoter polymorphism in patients with gastroesophageal malignancy. Am J Clin Nutr. 2009; 89(4): 1164-1172.

21. Sun F, Sun Y, Zhang D, Zhang J, Song B, Zheng H. Association of interleukin-10 gene polymorphism with cachexia in Chinese patients with gastric cancer. Ann Clin Lab Sci. 2010; 40(2): 149-155.

22. Larsson L, Rymo L, Berglundh T. Sp1 binds to the G allele of the- 1087 polymorphism in the IL-10 promoter and promotes IL-10 mRNA transcription and protein production. Genes Immun. 2010; 11(2): 181-187.

23. Deans C, Rose-Zerilli M, Wigmore S, et al. Host cytokine genotype is related to adverse prognosis and systemic inflammation in gastro-oesophageal cancer. Ann Surg Oncol. 2007; 14(2): 329-339. 